\title{
Correlation between the promoter methylation status of ATP-binding cassette sub-family $G$ member 2 and drug sensitivity in colorectal cancer cell lines
}

\author{
HYUN-HYE MOON ${ }^{1 *}$, SUNG-HEE KIM ${ }^{1 *}$ and JA-LOK KU ${ }^{1,2}$ \\ ${ }^{1}$ Laboratory of Cell Biology, Cancer Research Institute and ${ }^{2}$ Department of Biomedical Sciences, \\ Seoul National University College of Medicine, Seoul 110-799, Republic of Korea
}

Received June 30, 2015; Accepted August 10, 2015

DOI: $10.3892 /$ or.2015.4342

\begin{abstract}
Resistance to chemotherapeutic agents has been considered as a major reason for the high incidence rate of recurrence and metastasis suffered by colorectal cancer (CRC) patients. ATP-binding cassette sub-family $G$ member 2 $(A B C G 2)$ is involved in drug resistance. DNA methylation of the $A B C G 2$ promoter site has a significant influence on the regulation of epigenetic gene expression. In the present study, we investigated whether the methylation status of the $A B C G 2$ promoter is related to drug sensitivity in CRC cell lines. In order to examine the $A B C G 2$ expression level and identify the methylation status, RT-PCR, qRT-PCR analysis, MS-PCR and bisulfite sequencing were conducted on $32 \mathrm{CRC}$ cell lines. SNU-C4, LS174T and NCI-H716 were selected as low $A B C G 2$-expressing and high promoter methylated cell lines. The cell proliferation assay for 5-fluorouracil, oxaliplatin and irinotecan was performed after 5-aza-2'-deoxycytidine (5-aza) treatment in these cell lines. In the $32 \mathrm{CRC}$ cell lines, $25 \%$ of the cell lines expressed low or no $A B C G 2$ expression. Of these cell lines, SNU-C4, LS174T and NCI-H716 were hypermethylated at the promoter region, $20 \%$. Demethylation of $A B C G 2$ was induced by 5-aza, which enhanced the $A B C G 2$ expression level and influenced the cell proliferation similar to treatment with the anticancer agents. Our data suggest that the $A B C G 2$ expression level regulated by methylation is related to anticancer drug sensitivity. Based on these results, it can be applied to predict the anticancer drug response.
\end{abstract}

Correspondence to: Dr Ja-Lok Ku, Laboratory of Cell Biology, Cancer Research Institute, Seoul National University College of Medicine, 103 Daehak-ro, Jongno-gu, Seoul 110-799, Republic of Korea

E-mail:kujalok@snu.ac.kr

${ }^{*}$ Contributed equally

Key words: colorectal cancer, $A B C G 2$, promoter methylation, drug resistance

\section{Introduction}

Colorectal cancer (CRC) is the second most commonly diagnosed cancer in women and the third in men worldwide, with over 1.2 million new cancer cases and 608,700 deaths estimated to have occurred in 2008 (1). Although it is possible to cure colon cancer by surgery, the cure rate is moderate to poor depending on the stage of the cancer (2). Patients with stage II and III colorectal cancers remain at a high risk for tumor recurrence after curative resection. Therefore, they may benefit from additional treatment including adjuvant therapy. Chemotherapy and radiotherapy have been mainly used as an initial treatment to shrink any cancer and then commonly surgery is carried out to remove any tumors. During chemotherapy, a significant obstacle to the successful treatment of CRC patients is intrinsic or acquired drug resistance in patients who initially respond to chemotherapy.

Many mechanisms of drug resistance such as amplification or mutation of drug target genes, hypoxia, heterogeneity of cell subpopulations and defective drug transport or overexpression of p170 (protein of multidrug resistance), have been identified and studied using principally tumor cell lines $(3,4)$. A major mechanism of drug resistance in vitro is the overexpression of energy-dependent drug efflux pumps known as the ATP-binding cassette (ABC) superfamily including P-glycoprotein (MDR1), the multidrug resistance protein (MRP) and ATP-binding cassette sub-family $G$ member 2 (ABCG2) (5). They transport various compounds such as lipids, bile acids, xenobiotics and peptides for antigen presentation $(6,7)$.

$A B C G 2$, otherwise known as breast cancer resistance protein (BCRP) and mitoxantrone-resistant associated gene (MXR), was identified in high mitoxantrone-resistant-MCF-7/AdrVp and human colon cancer cell line, S1-M1-80. ABCG2 contains a 655-amino acid polypeptide transporter with six transmembrane domains and forms a homodimer. Additionally, the ABCG2 protein was reported to be a $72-\mathrm{kDa}$ protein. As a half transporter, two nucleotide binding proteins are required to perform as a drug efflux pump (8,9). ABCG2 expression is regulated by a TATA-less promoter which contains several SP1, AP1 and AP2 sites and putative $\mathrm{CpG}$ islands. It has been noted that the potential 
$\mathrm{CpG}$ islands in the promoter site may be regulated by methylation (7). Furthermore, the 5 ' region upstream of the basal promoter was revealed as both a positive and negative regulatory domain $(7,10)$.

$A B C G 2$ expression has been shown to be upregulated in some renal clear cell carcinomas and lung cancer, breast cancer and multiple myeloma cell lines after treatment with 5-aza-2'-deoxycytidine (5-aza), a DNA demethylating agent (7,11-14). Therefore, this observation suggested that the DNA methylation of the $A B C G 2$ promoter site, which consists of many $\mathrm{CpG}$ islands, could play a role in the epigenetic regulation of gene expression (10).

$A B C G 2$ causes resistance to certain chemotherapeutic drugs such as mitoxantrone, doxorubicin and daunorubicin in breast cancers by releasing its substrates which include topoisomerase I and II inhibitors (15). Furthermore, overexpression of $A B C G 2$ was found in drug-selected cell lines from breast, colon, gastric, lung and ovary cancers (16). In a study using non-small cell lung cancer (NSCLC), the chemotherapeutic response rate in patients was found to be correlated with $A B C G 2$ expression (17). In addition, 5-FU resistance was increased in $A B C G 2$-transfected MDCKII cells (18). Thus, drug resistance might be induced by the regulation of $A B C G 2$ expression in 5-FU, irinotecan and oxaliplatin-resistant cell lines. To investigate whether the $A B C G 2$ expression level and methylation status of the promoter affect drug sensitivity in CRC cell lines, we investigated the expression pattern of $A B C G 2$ and the methylation status of the $A B C G 2$ promoter.

To show that $A B C G 2$ expression is regulated by promoter methylation in CRC cell lines, we analyzed the mRNA expression of $A B C G 2$ and methylation status of the $A B C G 2$ promoter in $32 \mathrm{CRC}$ cell lines. Afterwards, we studied whether $A B C G 2$ expression and methylation status have an influence on anticancer drug sensitivity using the cell proliferation assay, WST-1 assay. Since drug sensitivity increased in several demethylated CRC cell lines, the results of this study suggest that DNA methylation of $A B C G 2$ can be a drug resistance marker for $\mathrm{CRC}$ patients who have resistance to chemotherapeutic drugs.

\section{Materials and methods}

Cell culture. The 32 CRC cell lines were provided by the Korean Cell Line Bank (KCLB, Seoul, Korea). All cell lines were cultured in RPMI-1640 medium except for Caco-2 and WiDr. Caco-2 was maintained in minimum essential medium and Dulbecco's modified Eagle's medium was used for WiDr. Each medium was supplemented with $10 \%$ fetal bovine serum and $1.1 \%$ penicillin/streptomycin. Cells were incubated in humidified incubators at $37^{\circ} \mathrm{C}$ with $5 \% \mathrm{CO}_{2}$ and $95 \%$ air.

Genomic DNA extraction. Genomic DNA was extracted from the $32 \mathrm{CRC}$ cell lines using the G-DEX ${ }^{\mathrm{TM}}$ IIc genomic DNA extraction kit (Intron Biotechnology, Gyeonggi, Korea) following the manufacturer's instructions. Cells treated with trypsin were collected and then suspended in cell lysis buffer. RNase A solution was added to the cell lysates and they were incubated at $37^{\circ} \mathrm{C}$. The protein precipitation step was carried out by adding PPT buffer, vortexing and then centrifuging the samples. The supernatant, which included the DNA, was collected and inverted with 2-propanol and then, the mixture was centrifuged at 13,000 rpm. The DNA pellet was dissolved in DNA rehydration buffer after washing with $70 \%$ ethanol.

RNA isolation and cDNA synthesis. Cells were collected with trypsinization and suspended in easy-BLUE ${ }^{\mathrm{TM}}$ (Intron Biotechnology). Total RNA was isolated according to the manufacturer's instructions. For cDNA synthesis, the QuantiTect Reverse Transcription kit (Qiagen, Venlo, The Netherlands) was used. The mixture was composed of $1 \mu \mathrm{g}$ of total RNA, $2 \mu 1$ gDNA wipe buffer and diethylpyrocarbonate (DEPC) water to make a mixture with volume $\leq 14 \mu \mathrm{l}$. After incubation at $42^{\circ} \mathrm{C}$ for $2 \mathrm{~min}, 4 \mu \mathrm{l}$ of RT buffer, $1 \mu \mathrm{l}$ of the RT primer mix and $1 \mu \mathrm{l}$ of RTase were mixed together and incubated at $42^{\circ} \mathrm{C}$ for $45 \mathrm{~min}$. The final reaction mixture was maintained at $95^{\circ} \mathrm{C}$ for $2 \mathrm{~min}$.

Bisulfite modification of genomic DNA. For bisulfite modification, $2 \mu \mathrm{g}$ of genomic DNA from the $32 \mathrm{CRC}$ cell lines were required. Bisulfite modification was processed using the EZ DNA Methylation ${ }^{\mathrm{TM}}$ kit (Zymo Research, Orange, CA, USA) following the manufacturer's instructions.

Reverse transcriptase-PCR (RT-PCR). To analyze the $A B C G 2$ mRNA expression level, $1 \mu 1$ of synthesized cDNA was amplified in a $14 \mu \mathrm{l}$ PCR mixture that contained 10X PCR buffer (with $\mathrm{MgCl}_{2}$ ), dNTPs, forward and reverse primers $(10 \mathrm{pmol} / \mu \mathrm{l})$ (Table I), distilled water and i-Taq DNA polymerase (Intron Biotechnology). The RT-PCR conditions consisted of $5 \mathrm{~min}$ at $94^{\circ} \mathrm{C}$ for an initial denaturation, followed by 35 cycles of $94^{\circ} \mathrm{C}$ for $30 \mathrm{sec}, 65^{\circ} \mathrm{C}$ for $1 \mathrm{~min}$, and $72^{\circ} \mathrm{C}$ for $30 \mathrm{sec}$ and a final elongation of $7 \mathrm{~min}$ at $72^{\circ} \mathrm{C}$. The reaction was carried out using a programmable thermal cycler (PCR System 9700, Applied Biosystems, Foster City, CA, USA). The PCR products were fractionated on a $1.5 \%$ agarose gel containing ethidium bromide.

Quantitative real-time PCR ( $q R T-P C R$ ). qRT-PCR was performed in a 386-well PCR plate containing SYBR-Green Master Mix (Applied Biosystems), distilled water, $10 \mathrm{ng}$ of the cDNA templates and $900 \mathrm{nM}$ of the $A B C G 2$ forward and reverse primers (Table I) (19). qRT-PCR analysis was performed with the 7900HT Fast Real-Time PCR system (Life Technologies Co, Carlsbad, CA, USA). The results were normalized to the housekeeping gene, $\beta$-actin, and the cycle threshold $(\mathrm{Ct})$ values were determined. This experiment was repeated three times.

Methylation-specific PCR (MS-PCR). The PCR reactions were performed at $94^{\circ} \mathrm{C}$ for $5 \mathrm{~min}$, and then 45 cycles of $94^{\circ} \mathrm{C}$ for $30 \mathrm{sec}$, $53^{\circ} \mathrm{C}$ for $1 \mathrm{~min}$ for the methylated region and $54^{\circ} \mathrm{C}$ for $1 \mathrm{~min}$ for the unmethylated region, and $72^{\circ} \mathrm{C}$ for $30 \mathrm{sec}$, and finally $72^{\circ} \mathrm{C}$ for $7 \mathrm{~min}$ for both PCR reactions. To analyze the methylation of the $A B C G 2$ promoter region, $1 \mu \mathrm{l}$ bisulfite modified DNA was amplified in a PCR mixture that contained 10X PCR buffer, dNTPs, forward and reverse primers for methylated or unmethylated DNA (10 pmol $/ \mu \mathrm{l})$ (Table I), 5X Q-solution, distilled water and Taq DNA polymerase (Qiagen).

Bisulfite sequencing analysis. The specific primers for the bisulfite sequencing analysis were designed using MethPrimer 
Table I. Primer sequences for RT-PCR, qRT-PCR, MS-PCR and bisulfite sequencing PCR.

\begin{tabular}{llcc}
\hline Name & \multicolumn{1}{c}{ Sequences } & Size (bp) & Refs. \\
\hline MXR RT F & 5'-GTTTATCCGTGGTGTGTCTGG-3' & 652 & \\
MXR RT R & 5'-CTGAGCTATAGAGGCCTGGG-3' & & $(19)$ \\
$A B C G 2$ qRT F & 5'-CAGGTCTGTTGGTCAATCTCACA-3' & 76 & \\
$A B C G 2$ qRT R & 5'-TCCATATCGTGGAATGCTGAAG-3' & & MethPrimer software \\
$A B C G 2$ M F & 5'-TATTTATTTAATTTGTTTTGGGTGC-3' & \multirow{2}{*}{141} & \\
$A B C G 2$ M R & 5'-TCATTAAACTAATCAATACCTCGTC-3' & & \\
$A B C G 2$ U F & 5'-TTTATTTAATTTGTTTTGGGTGTGA-3' & 139 & \\
$A B C G 2$ U R & 5'-TCATTAAACTAATCAATACCTCATC-3' & & \\
$A B C G 2$ BS F & 5'-AAATTATTTATTTAATTTGTTTTGG-3' & 282 & \\
$A B C G 2$ BS R & 5'-CCAACAAAACTAATACCACC-3' & & \\
\hline
\end{tabular}

F, forward; R, reverse; RT, RT-PCR-specific primer; qRT, qRT-PCR-specific primer; M, methylation-specific primer; U, unmethylation specific primer; BS, bisulfite sequencing-specific primer. MethPrimer software (www.urogene.org/ methprimer/index 1.html).
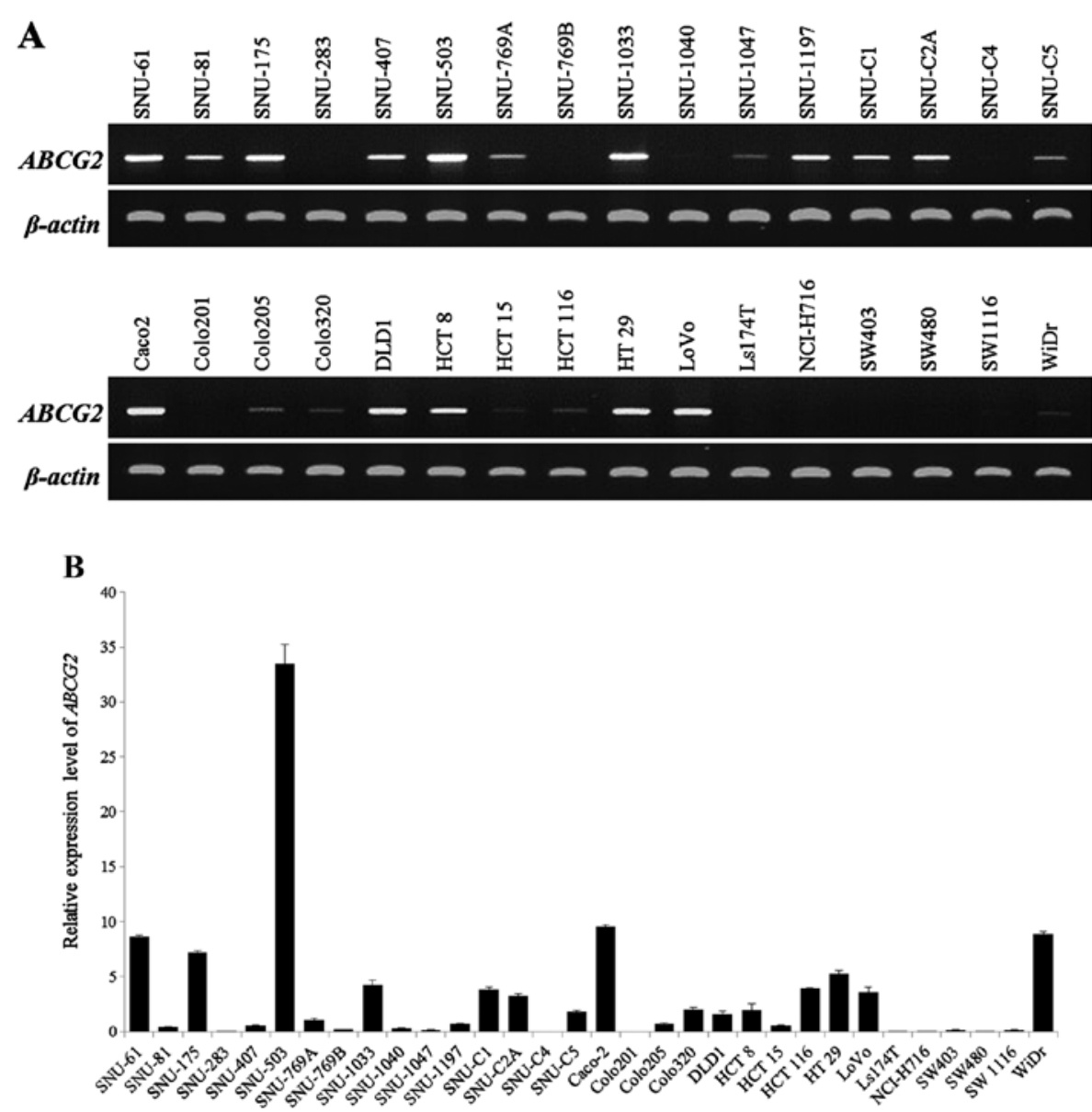

Figure 1. Expression analysis of the $A B C G 2$ gene was performed in 32 colorectal cancer cell lines by RT-PCR and qRT-PCR. (A) RT-PCR analysis for screening the $A B C G 2$ mRNA expression level in 32 colorectal cancer cell lines. ABCG2 expression was shown in 23 cell lines (SNU-61, SNU-81, SNU-175, SNU-407, SNU-503, SNU-769A, SNU-1033, SNU-1047, SNU-1197, SNU-C1, SNU-C2A, SNU-C5, Caco-2, Colo205, Colo320, DLD1, HCT 8, HCT 15, HCT 116, HT 29, LoVo, SW1116 and WiDr) but not in 9 cell lines (SNU-283, SNU-769B, SNU-1040, SNU-C4, Colo201, LS174T, NCI-H716, SW403 and SW480). (B) Quantitative differences in ABCG2 mRNA expression as determined by qRT-PCR analysis.

software (http://www.urogene.org/methprimer/index1. html) (Table I). The PCR reaction was carried out at $94^{\circ} \mathrm{C}$ for $5 \mathrm{~min}$, with 40 amplification cycles of $94^{\circ} \mathrm{C}$ for $30 \mathrm{sec}, 52^{\circ} \mathrm{C}$ for $1 \mathrm{~min}$ and $72^{\circ} \mathrm{C}$ for $30 \mathrm{sec}$ with a final extension step at $72^{\circ} \mathrm{C}$ for $7 \mathrm{~min}$. The amplicons from the bisulfite sequencing primers were inserted into the pGEM-T Easy vector (Promega, 
Madison, WI, USA) for TA-cloning. Sequences from five individual colonies for each $\mathrm{CRC}$ cell line were sequenced using universal pUC/M13 primers and each sequence was analyzed using a Taq dideoxy terminator cycle sequencing kit on an ABI 3730 DNA sequencer (Applied Biosystems).

5-aza-2'-deoxycytidine treatment. For treatment with 5-aza, $2 \times 10^{5}$ cells $/ \mathrm{ml}$ were seeded in two $75 \mathrm{~cm}^{2}$ culture flasks. On the following day, one of the flasks was treated with $3 \mu \mathrm{M}$ of 5-aza (Sigma-Aldrich) and the other flask received the same volume of DMSO as an untreated group for a 48-h incubation time.

Cell proliferation assay. Cells were seeded on a 96 -well plate at $2 \times 10^{4}$ cells/well and incubated overnight at $37^{\circ} \mathrm{C}$ in $5 \% \mathrm{CO}_{2}$ and $95 \%$ air. On the following day, anticancer drugs including 5-FU, irinotecan and oxaliplatin (all from Sigma-Aldrich) were added separately into the well at $48 \mathrm{~h}$ after 5-aza treatment. Cell proliferation reagent EZ-Cytox (DoGen, Seoul, Korea) was added to each well after a 72-h incubation time from the addition of the anticancer drugs. Then, the plates were incubated at $37^{\circ} \mathrm{C}$ for $4 \mathrm{~h}$, and the absorbance was measured with a Multiscan FC microplate photometer (Thermo Scientific Inc., Bremen, Germany) at $450 \mathrm{~nm}$. This assay was performed in triplicate wells.

Statistical analysis. Numerical data for all graphs are expressed as the mean \pm standard deviation (SD). $\mathrm{P}<0.05$ was considered to indicate a statistically significant difference, and statistical analysis was carried out with SPSS software version 20.0.

\section{Results}

Expression of $A B C G 2$ in the $C R C$ cell lines. CRC cell lines were examined by RT-PCR and qRT-PCR to identify the mRNA expression level of $A B C G 2$. After gel electrophoresis, obtained RT-PCR bands were processed by ImageJ (http://rsbweb. nih.gov/ij/) as the rate of $A B C G 2$ expression using the formula: $A B C G 2$ expression = (amplified $A B C G 2 /$ amplified $\beta$-actin) x 100 . The $A B C G 2$ mRNA band was detected in 23 cell lines (range of expression rate from 2.4 to 116.7, data not shown) but not in 9 cell lines (Fig. 1A and Table II). Additionally, we classified the groups into high ( $>1$ of the relative expression level) and low ( $<1$ of relative expression level) groups according to the relative expression level shown by qRT-PCR (Fig. 1B) [Relative expression level = $(A B C G 2$ expression level/ $\beta$-actin expression level) x 100; Table II]. SNU-61, SNU-175, SNU-503, SNU-769A, SNU-1033, SNU-C1, SNU-C2A, SNU-C5, Caco-2, DLD1, Colo320, HCT 8, HCT 116, HT 29, LoVo and WiDr showed a relatively higher mRNA expression level of $A B C G 2$ and SNU-81, SNU-283, SNU-407, SNU-769B, SNU-1047, SNU-1197, SNU-C4, Colo201, Colo205, HCT 15, LS174T, NCI-H716, SW403, SW480 and SW1116 cell lines belonged to the low group. The relative expression level was not detected in 4 cell lines: SNU-C4, Colo201, LS174T and SW480. Taken together, there were 8 cell lines that had low or no mRNA expression for $A B C G 2$ in the RT-PCR and qRT-PCR analyses: SNU-283, SNU-769B, SNU-C4, Colo201, LS174T, NCI-H716, SW403 and SW480.
Table II. Correlation between the promoter methylation status and $A B C G 2$ expression.

\begin{tabular}{|c|c|c|c|c|}
\hline $\begin{array}{l}\text { Cell } \\
\text { lines }\end{array}$ & Methylation & Unmethylation & $\begin{array}{c}\text { Expression } \\
(\%)\end{array}$ & $\begin{array}{c}\text { Methylation } \\
(\%)\end{array}$ \\
\hline SNU-61 & + & - & 8.7 & 1.9 \\
\hline SNU-81 & + & + & 0.4 & 0.0 \\
\hline SNU-175 & + & + & 7.2 & 3.8 \\
\hline SNU-283 & - & - & 0.1 & 14.3 \\
\hline SNU-407 & + & + & 0.5 & 2.9 \\
\hline SNU-503 & + & + & 33.5 & 2.9 \\
\hline SNU-769A & + & + & 1.0 & 57.1 \\
\hline SNU-769B & + & + & 0.2 & 0.0 \\
\hline SNU-1033 & + & - & 4.3 & 0.0 \\
\hline SNU-1040 & - & - & 0.3 & 0.0 \\
\hline SNU-1047 & + & + & 0.2 & 0.0 \\
\hline SNU-1197 & + & + & 0.7 & 0.0 \\
\hline SNU-C1 & + & - & 3.8 & 7.6 \\
\hline SNU-C2A & + & + & 3.3 & 5.7 \\
\hline SNU-C4 & + & + & 0.0 & 28.6 \\
\hline SNU-C5 & + & - & 1.8 & 1.9 \\
\hline Caco-2 & + & - & 9.6 & 0.0 \\
\hline Colo201 & + & - & 0.0 & 2.9 \\
\hline Colo205 & + & - & 0.7 & 19.0 \\
\hline Colo320 & + & - & 2.0 & 17.1 \\
\hline DLD1 & + & - & 1.6 & 0.0 \\
\hline НCT 8 & + & + & 2.0 & 0.0 \\
\hline HCT 15 & + & + & 0.6 & 1.0 \\
\hline HCT 116 & + & + & 3.9 & 1.0 \\
\hline HT 29 & + & - & 5.3 & 0.0 \\
\hline LoVo & + & - & 3.6 & 30.5 \\
\hline LS174T & + & + & 0.0 & 24.8 \\
\hline NCI-H716 & + & + & 0.1 & 45.7 \\
\hline SW403 & + & - & 0.2 & 1.0 \\
\hline SW480 & + & + & 0.0 & 4.8 \\
\hline SW1116 & + & - & 0.1 & 4.8 \\
\hline WiDr & + & - & 8.9 & 0.0 \\
\hline
\end{tabular}

-, PCR bands were not detected; +, PCR bands were detected; $\%$ methylation level of methylated CpGs [(No. of methylated CpGs/no. of total CpGs) x 100]; \% expression, relative expression level of $A B C G 2$ as a ratio to the $\beta$-actin expression level [( $A B C G 2$ level/ $\beta$-actin level) $\mathrm{x} 100]$.

Evaluation of the promoter methylation status of the ABCG2 gene by MS-PCR and bisulfite sequencing analysis. To determine whether $A B C G 2$ expression is related to epigenetic changes such as $\mathrm{CpG}$ methylation of the promoter site, we investigated the methylation status of the $A B C G 2$ promoter site in 32 CRC cell lines with MS-PCR and bisulfite sequencing analysis. Genomic DNA, which was modified with sodium bisulfite, had all unmethylated cytosines converted to uracils but methylated cytosines remained unchanged. The specifically designed primers (Table I) for MS-PCR amplified the unmethylated and methylated sequences located from -273 to -414 which contained $21 \mathrm{CpG}$ islands (Fig. 2 and Table III). 

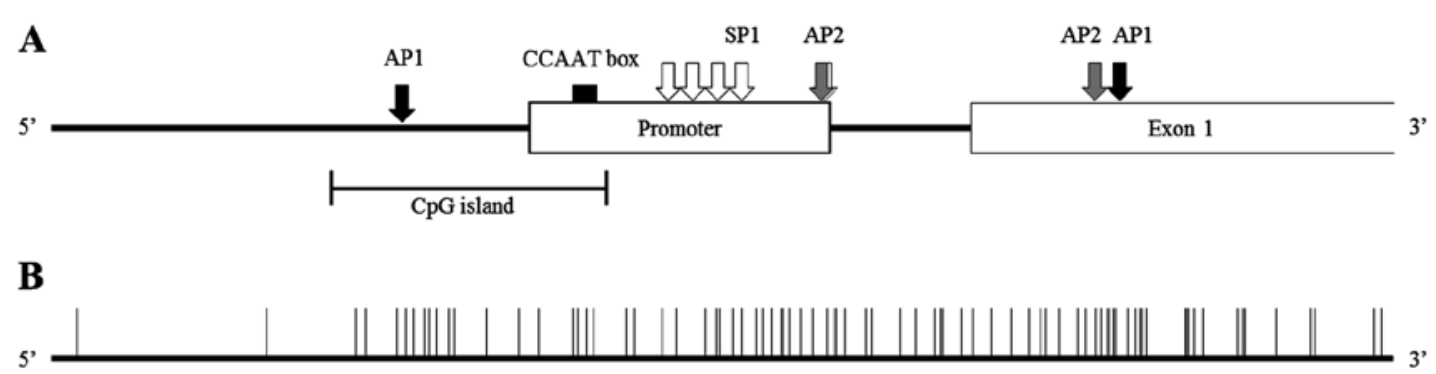

Figure 2. Schemes of $\mathrm{CpG}$ island in the promoter region of $A B C G 2$. (A) Scheme of the $A B C G 2$ gene 5' upstream region. CpG island (-402 to -249), AP1 (black arrows), AP2 (grey arrow) SP1 (white arrows) and CCAAT box are included in this region (7,11). (B) Genomic map of the CpG dinucleotide sites. Horizontal bars indicate introns and vertical bars indicate the distribution of the $A B C G 2 \mathrm{CpG}$ dinucleotides $(-592$ to +358$)$.

Table III. List of transcriptional regulation sites and genomic regions in $A B C G 2$.

\begin{tabular}{lc}
\hline Potential site & Genomic position \\
\hline Promoter site & -36 to -266 \\
XBBF & -363 to -378 \\
CpG island & -249 to -402 \\
SP1 site & -210 to -222 \\
& -178 to -187 \\
& -151 to -160 \\
& -116 to -127 \\
& -37 to -49 \\
AP1 site & -349 to -360 \\
CCAAT box & +124 to +136 \\
AP2 site & -275 to -280 \\
& -38 to -50 \\
Exon 1 & +107 to +118 \\
\hline
\end{tabular}

Nucleotide positions of the sites in the $A B C G 2$ genome are designated with the transcriptional start site $(+1)$, as determined in a previous study (6).

Methylated DNAs were detected in all cell lines except for SNU-283, and there was a weak methylated band in SNU-1040 (Fig. 3A). Unmethylated DNAs were amplified weakly in most of the cell lines except for SNU-283 and SNU-1040 which did not show any methylated DNA bands. There were 10 cell lines (SNU-769B, SNU-1047, SNU-C4, Colo201, HCT 15, LS174T, NCI-H716, SW403, SW480 and SW1116) that had low or no expression of $A B C G 2$ mRNA and methylated DNAs. The expression levels of both $A B C G 2$ mRNA and amplified methylated DNAs were observed in the other 20 cell lines. In SNU-C4, Colo201, LS174T and SW480, methylated bands were present but $A B C G 2$ gene expression was not detected in RT-PCR and qRT-PCR (Fig. 1) at the same time. The $\mathrm{CpG}$ island region (-136 to -417) that contains $21 \mathrm{CpG}$ dinucleotide sites (Fig. 2 and Table III) and part of the promoter for the $A B C G 2$ gene was amplified with a bisulfite sequencing specific primer set (Table I). Part of the $\mathrm{CpG}$ island sequence was determined in Fig. 3B. SNU-769A represented the methylated $\mathrm{CpG}$ dinucleotide sequence and Caco-2 represented the unmethylated sequence around the seven $\mathrm{CpG}$ islands. The methylation status of the $\mathrm{CpG}$ island in the $A B C G 2$ promoter is shown in Fig. 3C. To compare the $A B C G 2$ mRNA and methylation status of the promoter, the percentage of methylation was analyzed (Table II). The percentage of promoter methylation of $A B C G 2$ in 8 cell lines was $>10 \%$ and SNU-769A (57.1\%), NCI-H716 (45.7\%) and SNU-C4 (28.6\%) were verified as having a hypermethylated $A B C G 2$ promoter. SNU-C4, LS174T and NCI-H716 had $>20 \%$ methylation in the promoter and simultaneously low or no $A B C G 2$ gene expression was detected (Fig. 1).

Recovery of ABCG2 mRNA expression after treatment with 5-aza. To determine whether DNA methylation affects ABCG2 expression, we chosen three CRC cell lines (SNU-C4, LS174T and NCI-H716) that showed methylated DNAs in the MS-PCR, $>20 \%$ methylated $\mathrm{CpG}$ dinucleotides in the bisulfite sequencing analysis and weak or no $A B C G 2$ mRNA expression. In all three cell lines, $A B C G 2$ mRNA expression was recovered when the cell lines were cultured with $3 \mu \mathrm{M}$ of 5 -aza for $48 \mathrm{~h}$ (Fig. 4). Furthermore, there was no significant re-expression of $A B C G 2$ when the LS174T and NCI-H716 cell lines were treated with trichostatin A (TSA, histone deacetylase inhibitor) (data not shown). Therefore, re-expression of $A B C G 2$ mRNA resulted from demethylation mediated by 5 -aza, not acetylation.

Drug sensitivity is reversed by 5-aza treatment in several CRC cell lines. To determine whether mRNA re-expression by demethylation affects anticancer drug sensitivity, we performed the WST-1 assay using 5-aza-treated CRC cell lines which expressed a low mRNA level under relative expression level 1 and had $>20 \%$ methylation of the promoter (Table II). Selected cell lines, SNU-C4, LS174T and NCI-H716, were treated with chemotherapeutic drugs in a dose-dependent manner used to treat CRC patients known as $A B C G 2$ substrates: 5-FU, irinotecan and oxaliplatin. Drug sensitivity was measured inversely by cell viability depending on the absorbance at $450 \mathrm{~nm}$. In the SNU-C4, LS174T and NCI-H716 cell lines treated with 5-aza, the cell viability was significantly increased in the presence of 5-FU, irinotecan and oxaliplatin at all drug concentrations (Fig. 5).

5 -aza potentiated the cell viability together with 5 -FU (1.83-fold to 4.33 -fold increase with $10 \mu \mathrm{g}$ ), irinotecan (1.52-fold to 2.43 -fold increase with $200 \mu \mathrm{M})$ and 

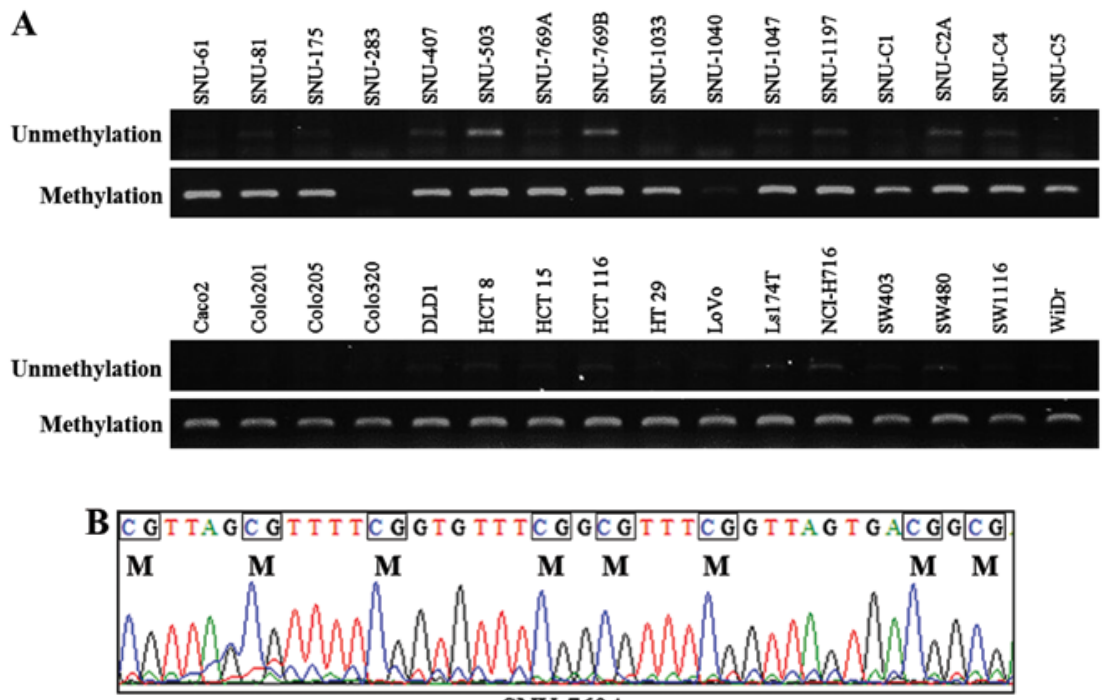

SNU-769A

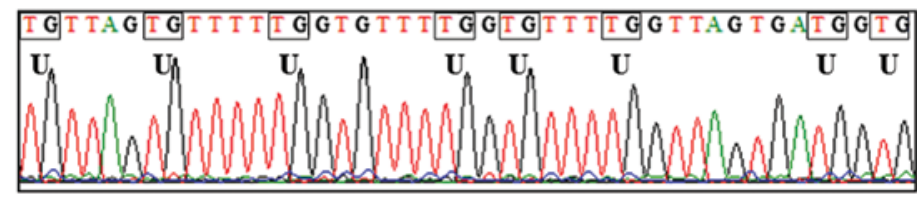

Caco2
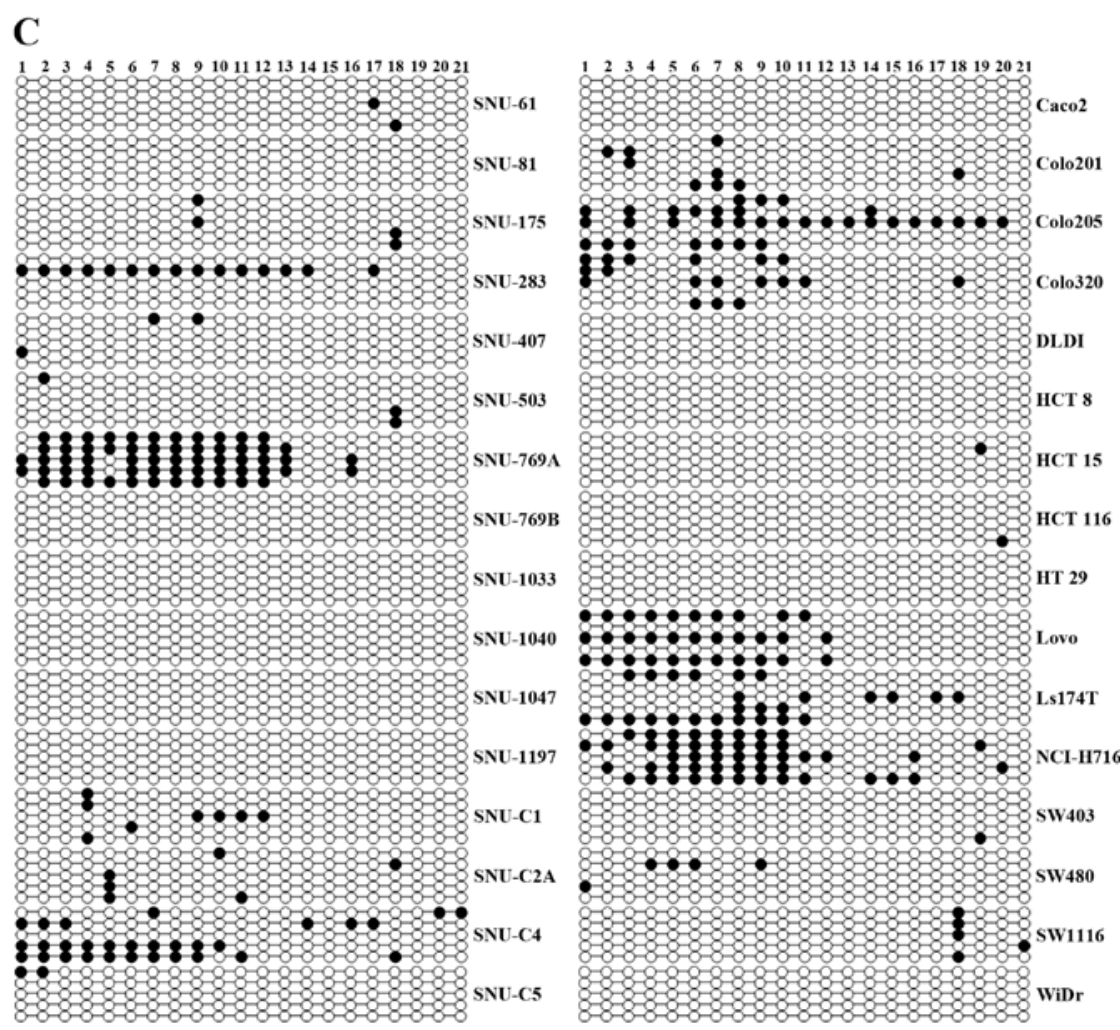

Figure 3. Methylation status of the $A B C G 2$ gene is shown in 32 colorectal cancer cell lines by MS-PCR and bisulfite sequencing analysis. gDNA of the examined cell lines was processed by bisulfite modification before each analysis. (A) Unmethylated and methylated products were amplified by primers that recognize methylated and unmethylated sequences. (B) Representative sequence diagrams of methylated DNA sequencing analysis in SNU-769A (methylated) and Caco-2 (unmethylated) cell lines. Boxes indicate $\mathrm{CpG}$ dinucleotide sites in $21 \mathrm{CpG}$ islands. M, methylated site; U, unmethylated site. (C) Bisulfite sequencing analysis of $21 \mathrm{CpG}$ dinucleotides. All circles represent $\mathrm{CpG}$ dinucleotides. Open circles are unmethylated CpGs and closed circles are methylated CpG dinucleotides.

oxaliplatin (1.48-fold to 1.62 -fold increase with 50 and $100 \mu \mathrm{M})$ in the SNU-C4 cell line. In LS174T with 5-aza, cell viability was maximally increased at $50 \mu \mathrm{g}$ of 5-FU (2.67-fold), $200 \mu \mathrm{M}$ of irinotecan (2.45-fold) and $25 \mu \mathrm{M}$ of oxaliplatin (2.18-fold). The cell viability of 5-aza-treated NCI-H716 cells reached the greatest level at $50 \mu \mathrm{g}$ of $5-\mathrm{FU}$ (1.40-fold), $200 \mu \mathrm{M}$ of irino- 
A

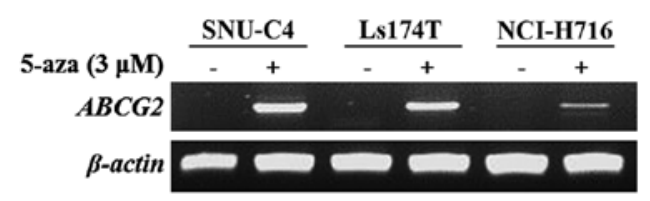

B

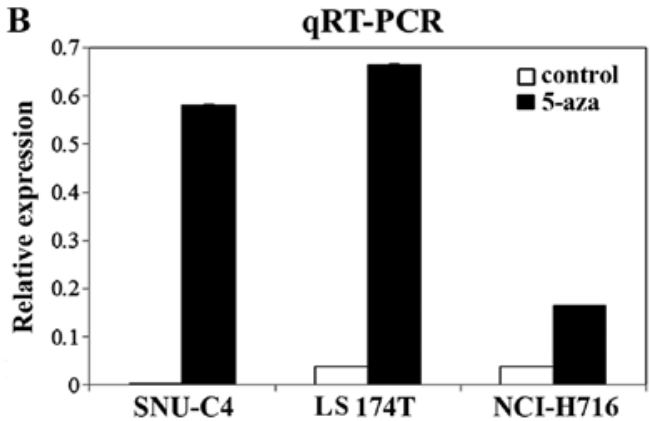

Figure 4. A comparison of the $A B C G 2$ mRNA expression level between 5-aza-treated and non-treated cell lines. We observed whether re-expression of $A B C G 2$ occurred in 3 cell lines after treatment with or without $3 \mu \mathrm{M}$ of 5 -aza. (A) RT-PCR. (B) qRT-PCR.
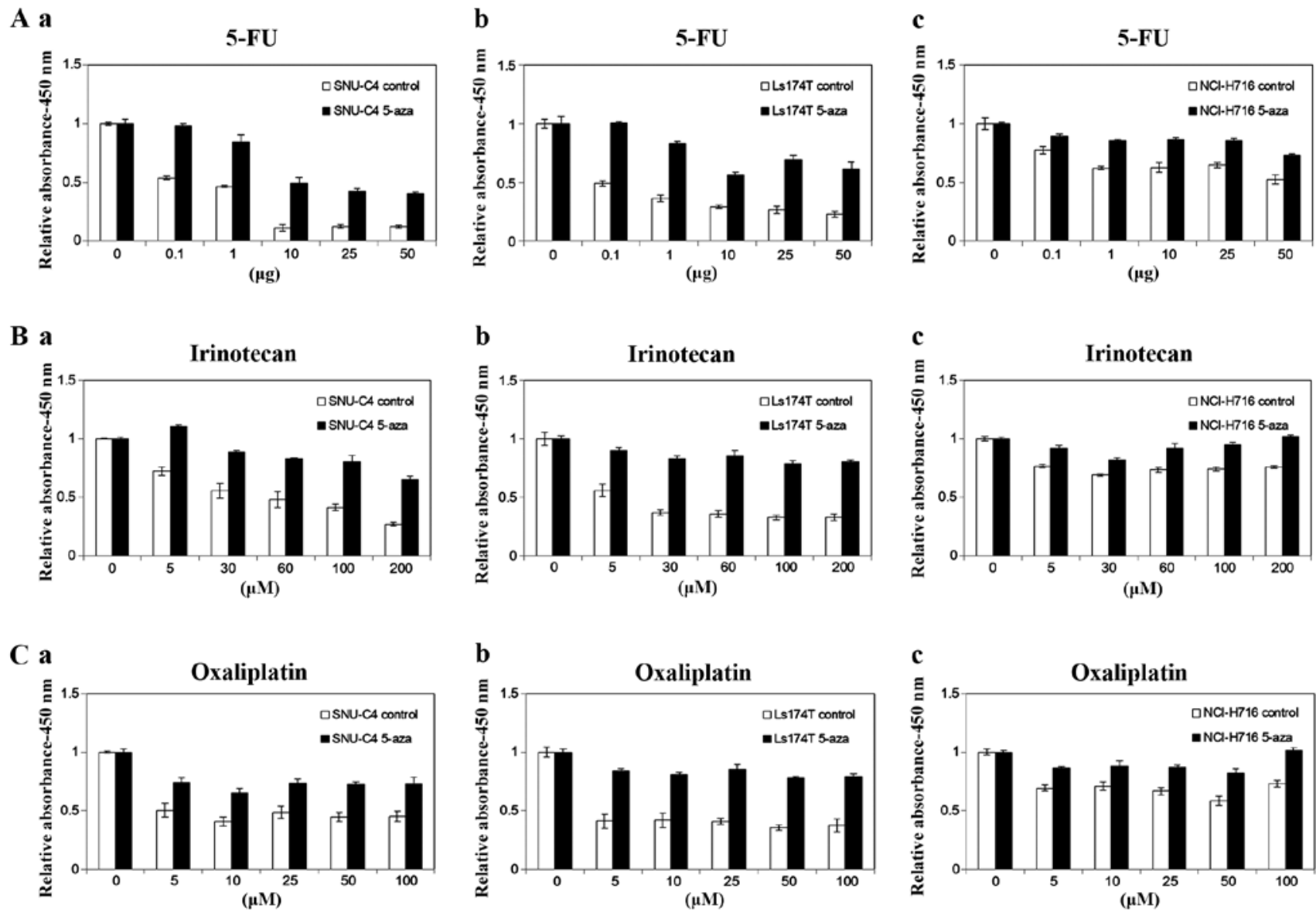

Figure 5. A comparison of cell viability for anticancer drugs in the colorectal cancer cell lines with or without 5-aza treatment. (a) SNU-C4, (b) LS174T and (c) NCI-H716 were treated with (A) 5-FU $(0,0.1,1,10,25$ and $50 \mu \mathrm{g})$, (B) irinotecan $(0,5,30,50,100$ and $200 \mu \mathrm{M})$ and $(\mathrm{C})$ oxaliplatin $(0,5,10,25,50$ and $100 \mu \mathrm{M})$ for $72 \mathrm{~h}$ after demethylation by 5 -aza, and cell viability was determined using WST-1 assay.

tecan (1.35-fold) and $50 \mu \mathrm{M}$ of oxaliplatin (1.40-fold). SNU-C4 (2.92-fold increase with 5-FU) and LS174T (2.22-fold increase with irinotecan and 2.08-fold increase with oxaliplatin) showed a maximum increase in cell viability for each anticancer drug, and a minimal increase was detected in the NCI-H716 cells (1.33-fold increase with 5-FU, 1.25-fold increase with irinotecan and 1.32-fold increase with oxaliplatin) according to the average cell viability. Additionally, increments in cell viability were observed at the greatest level when the cell lines were treated with 5-FU (2.18-fold) and oxaliplatin (1.65-fold) had the lowest level for the average enhanced cell viability. Taken together, 5-aza treatment which induces the demethylation of
$A B C G 2$ in several colorectal cell lines has an effect on the decrease in drug sensitivity.

\section{Discussion}

Studies have reported that overexpression of $A B C G 2$ is associated with anticancer drug resistance by mediating drug efflux. MCF-7/AdrVp cells are a multidrug-resistant human breast cancer subline which does not express P-gp or MRP1, known as multidrug resistance transporters, but does express $A B C G 2$. In this cell line, the multidrug resistance phenotype is acquired by $A B C G 2$ overexpression (15). The expression of $A B C G 2$ 
is regulated by DNA methylation, which has been known to be responsible for inhibiting gene expression. Methylation of the transcriptional regulatory region including the transcriptional binding sites induces the transcriptional repression of several genes $(20,21)$. In a prior study on lung cancer cells, it was discovered that methylation of the $A B C G 2$ promoter was inversely correlated with its expression (13). Following treatment with 5'-aza-2'-deoxycytidine, the DNA demethylation agent, $A B C G 2$ expression was re-activated. This indicated that DNA methylation of the promoter site, which consists of many $\mathrm{CpG}$ islands, could play a central role in the epigenetic regulation of $A B C G 2$ gene expression (11).

To study the correlation between the methylation patterns of the $A B C G 2$ promoter region and gene expression in CRC cell lines, we performed MS-PCR and bisulfite sequencing analysis. The $A B C G 2$ mRNA levels were examined by RT-PCR and quantitative real-time PCR. First, we classified the CRC cell lines into high or low $A B C G 2$ expression groups according to the relative expression level shown by qRT-PCR (Fig. 1B). The mean relative $A B C G 2$ expression value of the high group was $>2$. Then, we selected cell lines which had a hypermethylated promoter site identified by MS-PCR (Fig. 3A) and bisulfite sequencing analysis (Fig. 3C). As a result, SNU-C4, LS174T and NCI-H716 cells were selected as they exhibited low expression of the $A B C G 2$ gene less than the relative expression level 1 and had $>20 \%$ methylated $\mathrm{CpG}$ dinucleotides in the promoter site (Table II). The three cell lines were treated with demethylating agent 5-aza to determine whether DNA demethylation increases $A B C G 2$ mRNA expression. After treatment of the cell lines SNU-C4, LS174T and NCI-H716 with $3 \mu \mathrm{M} 5$-aza for $48 \mathrm{~h}, A B C G 2$ mRNA was re-expressed in all three cell lines (Fig. 4). Consequently, demethylation of the $\mathrm{CpG}$ dinucleotides in the $A B C G 2$ promoter upregulated $A B C G 2$ gene expression. In other words, the promoter was negatively regulated by DNA methylation in several CRC cell lines. However, we demonstrated that SNU-769A moderately expressed the $A B C G 2$ gene and had hypermethylation of promoter $\mathrm{CpG}$ islands (Table II). As referred to earlier in the study, 1 allele of the chromosome was methylated but another allele was not methylated in the moderate $A B C G 2$-expressing cells (NCI-H460, NCI-H441 and NCI-H358 cell lines) (13). Therefore, there is a possibility that 1 allele might be methylated in SNU-769A. However, to make sure of this speculation, additional DNA sequencing is required to analyze both alleles of SNU-769A. Additionally, there were somewhat different cases. For instance, in SNU-283 and SW480 cells, the ABCG2 mRNA was merely expressed and was not observed to be hypermethylation. In this case, we speculate that there are other pathways which regulate the expression of $A B C G 2$, such as histone acetylation or methylation. Further study is warranted to verify this speculation.

Various epigenetic modification types affect the regulation of genes such as acetylation at Lys and methylation at Arg and Lys. When the LS174T and NCI-H716 cell lines were treated with TSA, there was no significant re-expression of the $A B C G 2$ gene (data not shown). Taken together, these results suggested that methylation of the $A B C G 2$ promoter region might have an influence on $A B C G 2$ expression but acetylation might not be related to the regulation of the gene in various CRC cell lines. However, it is necessary to perform additional experiments such as the ChIP assay to determine whether other mechanisms or proteins are involved in the regulatory steps of $A B C G 2$ expression since methylation is not the only mechanism of epigenetic regulation.

In a previous study, it was shown that the development of drug resistance was not dependent on P-gp or MRP but was related to the upregulated protein expression of $A B C G 2$ in a mitoxantrone-resistant HT 29 colon carcinoma cell line (22). Likewise, $A B C G 2$ was overexpressed in irinotecan and oxaliplatin resistant cell lines, and 5-FU resistance was increased in $A B C G 2$-transfected MDCKII cells $(18,23)$. 5-FU, irinotecan and oxaliplatin are substrates for $A B C G 2$ (24). In summary, these studies suggest that overexpression of $A B C G 2$ contributes to drug resistance in cancer cells.

After we confirmed that demethylation can enhance $A B C G 2$ gene expression in the SNU-C4, LS174T and NCI-H716 cell lines, we investigated whether drug sensitivity can be affected by the increased $A B C G 2$ gene expression following 5-aza-induced demethylation. Cell viability was measured by WST-1 assay and inversely indicates drug sensitivity. SNU-C4, LS174T and NCI-H716 cells were treated with 5-FU, irinotecan and oxaliplatin for $72 \mathrm{~h}$ after a 48 -h treatment with 5-aza. The reversible effects of drug sensitivity appeared significantly in all cell lines treated with 5-aza (Fig. 5). 5-aza maximally potentiated the cell viability of 5-FU (4.33-fold at $10 \mu \mathrm{g})$ in SNU-C4 cells, irinotecan (2.45-fold at $200 \mu \mathrm{M})$ in LS174T cells and oxaliplatin (2.18-fold at $50 \mu \mathrm{M})$ in LS174T cells. In NCI-H716, a minimal increase was measured according to the average increased cell viability (1.33-fold for 5-FU, 1.25-fold for irinotecan and 1.32-fold for oxaliplatin). Since inverse cell viability is considered equivalent to drug sensitivity, we concluded that drug sensitivity was decreased in the 5-aza-treated CRC cell lines despite the differences in the increased levels of cell viability. The reason why there were differences in the increased levels of cell viability is thought to be due to distinctions in the expression level of the $A B C G 2$ mRNA in each cell line. Actually, the increments for the ratio of $A B C G 2$ expression in the 5-aza-treated cell lines were 1.66-fold in SNU-C4, 25.16-fold in LS174T and 6.89 -fold in NCI-H716 cells. Taken together, we found that the 5-aza-induced demethylation of the promoter site in some colorectal cell lines might have an effect on the decrease in drug sensitivity through the positive regulation of $A B C G 2$ mRNA expression based on various tests. According to the results, overexpression of the $A B C G 2$ gene as well as the $A B C G 2$ methylation status may be useful as a marker of drug resistance in CRC patients regarding those regimens, and it is possible to understand individual specific drug sensitivity for each CRC patient. Thus, the present study is meaningful in terms of anticancer treatment as appropriate therapy could be provided to CRC patients.

In conclusion, we identified how the promoter methylation status of $A B C G 2$ regulates pharmaceutical resistance in CRC cell lines. $A B C G 2$ plays a role in drug efflux in many types of cancers. We found that demethylation upregulated $A B C G 2$ gene expression and the enhanced expression was negatively correlated to anticancer drug sensitivity in various CRC cell lines. However, these findings should be verified through additional study concerning other epigenetic mechanisms or clinical trials. 


\section{Acknowledgements}

This study was supported by the Priority Research Center Program (2009-0093820) through a National Research Foundation of Korea grant funded by the MSIP.

\section{References}

1. Jemal A, Bray F, Center MM, Ferlay J, Ward E and Forman D Global cancer statistics. CA Cancer J Clin 61: 69-90, 2011.

2. André T, Boni C, Mounedji-Boudiaf L, Navarro M, Tabernero J, Hickish T, Topham C, Zaninelli M, Clingan P, Bridgewater J, et al; Multicenter International Study of Oxaliplatin/5-Fluorouracil/Leucovorin in the Adjuvant Treatment of Colon Cancer (MOSAIC) Investigators: Oxaliplatin, fluorouracil and leucovorin as adjuvant treatment for colon cancer. N Engl J Med 350: 2343-2351, 2004.

3. Violette S, Poulain L, Dussaulx E, Pepin D, Faussat AM, Chambaz J, Lacorte JM, Staedel C and Lesuffleur T: Resistance of colon cancer cells to long-term 5-fluorouracil exposure is correlated to the relative level of $\mathrm{Bcl}-2$ and $\mathrm{Bcl}-\mathrm{X}(\mathrm{L})$ in addition to Bax and p53 status. Int J Cancer 98: 498-504, 2002.

4. Cazin JL, Gosselin P, Cappelaere P, Robert J and Demaille A: Drug resistance in oncology: From concepts to applications J Cancer Res Clin Oncol 119: 76-86, 1992.

5. Gottesman MM: Mechanisms of cancer drug resistance. Annu Rev Med 53: 615-627, 2002.

6. Eijdems EW, De Haas M, Coco-Martin JM, Ottenheim CP Zaman GJ, Dauwerse HG, Breuning MH, Twentyman PR, Borst P and Baas F: Mechanisms of MRP over-expression in four human lung-cancer cell lines and analysis of the MRP amplicon. Int J Cancer 60: 676-684, 1995.

7. Bailey-Dell KJ, Hassel B, Doyle LA and Ross DD: Promoter characterization and genomic organization of the human breast cancer resistance protein (ATP-binding cassette transporter $G 2)$ gene. Biochim Biophys Acta 1520: 234-241, 2001.

8. Litman T, Brangi M, Hudson E, Fetsch P, Abati A, Ross DD, Miyake K, Resau JH and Bates SE: The multidrug-resistant phenotype associated with overexpression of the new ABC half-transporter, MXR (ABCG2). J Cell Sci 113: 2011-2021, 2000.

9. Xu J, Liu Y, Yang Y, Bates S and Zhang JT: Characterization of oligomeric human half-ABC transporter ATP-binding cassette G2. J Biol Chem 279: 19781-19789, 2004

10. Turner JG, Gump JL, Zhang C, Cook JM, Marchion D, Hazlehurst L, Munster P, Schell MJ, Dalton WS and Sullivan DM: $A B C G 2$ expression, function, and promoter methylation in human multiple myeloma. Blood 108: 3881-3889, 2006.

11. To KK, Zhan Z and Bates SE: Aberrant promoter methylation of the $A B C G 2$ gene in renal carcinoma. Mol Cell Biol 26 $8572-8585,2006$.

12. To KK, Zhan Z, Litman T and Bates SE: Regulation of $A B C G 2$ expression at the $3^{\prime}$ untranslated region of its mRNA through modulation of transcript stability and protein translation by a putative microRNA in the S1 colon cancer cell line. Mol Cell Biol 28: 5147-5161, 2008
13. Nakano H, Nakamura $Y$, Soda $H$, Kamikatahira $M$, Uchida K, Takasu M, Kitazaki T, Yamaguchi H, Nakatomi K, Yanagihara K, et al: Methylation status of breast cancer resistance protein detected by methylation-specific polymerase chain reaction analysis is correlated inversely with its expression in drug-resistant lung cancer cells. Cancer 112: 1122-1130, 2008.

14. Mirza S, Sharma G, Pandya P and Ralhan R: Demethylating agent 5-aza-2-deoxycytidine enhances susceptibility of breast cancer cells to anticancer agents. Mol Cell Biochem 342: 101-109, 2010

15. Doyle LA, Yang W, Abruzzo LV, Krogmann T, Gao Y, Rishi AK and Ross DD: A multidrug resistance transporter from human MCF-7 breast cancer cells. Proc Natl Acad Sci USA 95: 15665-15670, 1998.

16. Allen JD and Schinkel AH: Multidrug resistance and pharmacological protection mediated by the breast cancer resistance protein (BCRP/ABCG2). Mol Cancer Ther 1: 427-434, 2002.

17. Yoh K, Ishii G, Yokose T, Minegishi Y, Tsuta K, Goto K, Nishiwaki Y, Kodama T, Suga M and Ochiai A: Breast cancer resistance protein impacts clinical outcome in platinum-based chemotherapy for advanced non-small cell lung cancer. Clin Cancer Res 10: 1691-1697, 2004.

18. König SK, Herzog M, Theile D, Zembruski N, Haefeli WE and Weiss J: Impact of drug transporters on cellular resistance towards saquinavir and darunavir. J Antimicrob Chemother 65: 2319-2328, 2010

19. Langmann T, Mauerer R, Zahn A, Moehle C, Probst M, Stremmel W and Schmitz G: Real-time reverse transcription-PCR expression profiling of the complete human ATP-binding cassette transporter superfamily in various tissues. Clin Chem 49: 230-238, 2003

20. Hendrich B and Bird A: Identification and characterization of a family of mammalian methyl-CpG binding proteins. Mol Cell Biol 18: 6538-6547, 1998 .

21. Watt F and Molloy PL: Cytosine methylation prevents binding to DNA of a HeLa cell transcription factor required for optimal expression of the adenovirus major late promoter. Genes Dev 2: 1136-1143, 1988.

22. Perego P, De Cesare M, De Isabella P, Carenini N, Beggiolin G, Pezzoni G, Palumbo M, Tartaglia L, Pratesi G, Pisano C, et al: A novel 7-modified camptothecin analog overcomes breast cancer resistance protein-associated resistance in a mitoxantrone-selected colon carcinoma cell line. Cancer Res 61: 6034-6037, 2001.

23. Boyer J, McLean EG, Aroori S, Wilson P, McCulla A, Carey PD, Longley DB and Johnston PG: Characterization of p53 wild-type and null isogenic colorectal cancer cell lines resistant to 5-fluorouracil, oxaliplatin and irinotecan. Clin Cancer Res 10: 2158-2167, 2004.

24. Yuan J, Lv H, Peng B, Wang C, Yu Y and He Z: Role of BCRP as a biomarker for predicting resistance to 5-fluorouracil in breast cancer. Cancer Chemother Pharmacol 63: 1103-1110, 2009. 